\title{
Brillouin light scattering investigations of structured permalloy films
}

\author{
B. Hillebrands, C. Mathieu, M. Bauer, and S. O. Demokritov \\ Fachbereich Physik, Universität Kaiserslautern, 67663 Kaiserslautern, Germany
}

B. Bartenlian and C. Chappert

IEF, Université Paris-Sud, 91405 Orsay, France

\section{Decanini, F. Rousseaux, and F. Carcenac \\ L2M, 92225 Bagneux, France}

\begin{abstract}
The static and spin wave properties of regular square lattices of magnetic dots of $0.5-2 \mu \mathrm{m}$ dot diameter and 1-4 $\mu \mathrm{m}$ periodicity patterned in permalloy films have been investigated by Brillouin light scattering. The samples have been structured using x-ray lithography and ion beam etching. The Brillouin light scattering spectra reveal both surface and bulk spin wave modes. The spin wave frequencies can be well described taking into account the demagnetization factor of each single dot. For the samples with smallest dot separation of $0.1 \mu \mathrm{m}$ a fourfold in-plane magnetic anisotropy with the easy axis directed along the pattern diagonal is observed, indicating anisotropic coupling between the dots. (C) 1997 American Institute of Physics. [S0021-8979(97)59808-2]
\end{abstract}

Magnetic films patterned on the $\mu \mathrm{m}$ and sub- $\mu \mathrm{m}$ scale have received increased attention in the last few years due to their potential applications as future storage media and sensors. There remain many unresolved problems in the preparation and characterization of these structures, and therefore suitable preparation procedures and analysis tools are still under development. It is of particular importance to develop fabrication methods for magnetic islands with a very narrow distribution of the magnetic parameters and with well controlled coupling between the islands, which for many applications should be made negligibly small. In particular a precise control of magnetic anisotropies induced by the patterning process as well as by finite size effects is required. In this article we report on studies of regular square arrays of magnetic dots by Brillouin light scattering from spin wave excitations present in the dots. From the measured spin wave dispersion properties the uniformity of the magnetization distribution, demagnetizing effects, as well as coupling strengths between magnetic dots are obtained.

We have prepared regular square arrays of circularly shaped dots of permalloy $\left(\mathrm{Ni}_{80} \mathrm{Fe}_{20}\right)$ of $0.5-2 \mu \mathrm{m}$ diameter and 500 and $1000 \AA$ thickness. The films were prepared in ultrahigh vacuum onto $\mathrm{Si} / \mathrm{SiO}_{2}$ wafer substrates using an $e$-beam evaporator. The samples were patterned by synchrotron radiation based $\mathrm{x}$-ray lithography performed at the $\mathrm{L} 2 \mathrm{M}$ synchrotron station at the super ACO storage ring at LURE, Orsay, France using ion beam etching to transfer the patterns into the permalloy films. A subtractive process was used as described elsewhere. ${ }^{1}$ It involved a lift-off of a 60 -nm-thick aluminum film, which acts as a mask during the ion beam etching of the permalloy. On one film four arrays with dot diameters/lattice periods of $2 / 4,2 / 2.2,1 / 2,1 / 1.1$, and $0.5 / 1$ $\mu \mathrm{m}$ and an area of $1 \mathrm{~mm}^{2}$, and on a second film an array of $0.5 / 1 \mu \mathrm{m}$ and an area of $4 \mathrm{~mm}^{2}$ have been patterned (the $0.5 / 1 \mu \mathrm{m}$ samples consists of square dots). The samples were characterized by electron microscopy.

We have analyzed the dynamic properties of these structures, i.e., the spin wave excitation spectra, by Brillouin light scattering. ${ }^{2}$ This approach offers high sensitivity and easy determination of magnetic anisotropies and the coupling strengths between magnetic dots. In particular we can study anisotropic coupling between magnetic dots as follows. The samples consist of circular shaped islands on a square lattice. Within each island no in-plane anisotropy is present, as tested independently on an unpatterned area of the sample and on dot arrays with large dot separations. Any observed in-plane anisotropy, which is of the same symmetry as the dot lattice, i.e., of fourfold symmetry, can only be induced by coupling between the islands, and it is thus a measure of the coupling strength between the dots. Furthermore, spin wave excitations sense the magnetic properties on a length scale comparable to the spin wave wavelength of $\approx 2000 \AA$, which is smaller than the characteristic pattern dimensions.

The spin wave excitation of interest is the dipolar surface mode (Damon-Eshbach mode), which is sensitive to the saturation magnetization, to the external field, to anisotropy contributions, and in particular, to the demagnetizing factor. As noted above measurements of the anisotropy constants allow us to determine the coupling between the magnetic dots. The dispersion equation is given for zero anisotropy by $^{3}$

$$
\nu=\gamma \cdot\left[H_{i} \cdot\left(H_{i}+4 \pi M_{s}\right)+\left(2 \pi M_{s}\right)^{2} \cdot\left(1-e^{-2 q_{\|} d}\right)\right]^{1 / 2}
$$

with $\gamma$ the gyromagnetic ratio, $M_{s}$ the saturation magnetization, $q_{\|}$the in-plane wave vector, and $d$ the dot thickness. $H_{i}$ is the internal field, which in absence of anisotropy is given by:

$$
H_{i}=H-N \cdot 4 \pi M_{s}
$$

with $N$ the demagnetizing factor of the magnetic dots. In Eq. (1) it is assumed, that the magnetization is saturated in the plane of the dots and that the product of dot diameter and $q_{\|}$is large. This is fulfilled for the investigated samples. For nonzero anisotropy the dispersion relation can be obtained only numerically. ${ }^{4}$

The Brillouin light scattering experiments have been performed in backscattering geometry using $\mathrm{Ar}^{+}$-ion laser light of wavelength $514.5 \mathrm{~nm}$ with an incident power of 100 $\mathrm{mW}$, and a laser focus size of $\approx 40 \mu \mathrm{m}$ in diameter, which is 


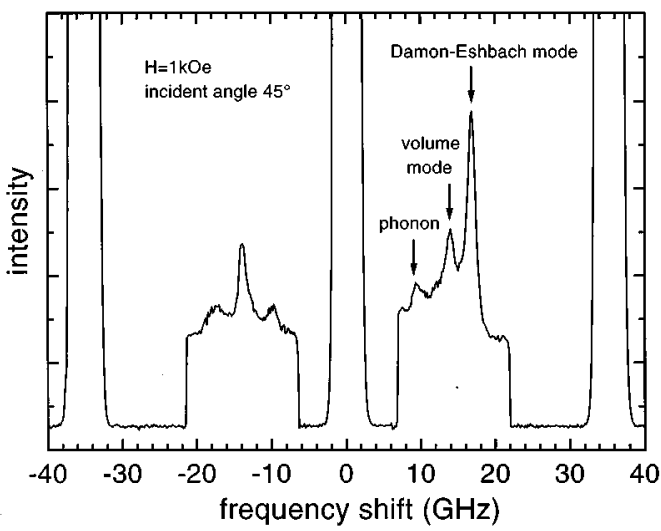

FIG. 1. Spin wave spectrum of a $2 / 4 \mu \mathrm{m}$ sample of $500 \AA$ thickness at an applied field of $1 \mathrm{kOe}$ at an angle of light incidence of $45^{\circ}$. At zero frequency the elastically scattered light, and at frequencies of $\pm(33-37) \mathrm{GHz}$ the first ghost peaks are observed. In the region of interest $\pm(6.5-22) \mathrm{GHz}$ the scan speed is reduced and thus the scattering intensity is increased. As indicated in the figure the Damon-Eshbach mode, a standing spin wave (volume mode) and a surface phonon is observed.

small enough compared to the patterned area. The applied magnetic field is oriented perpendicular to the scattering plane. The spectra have been recorded as a function of the directions and moduli of the in-plane wave vector, $q_{\|}$, and the external field, $H$, using a fully automated, computer controlled (3+3)-pass Sandercock-type tandem Fabry-Perot interferometer. In the regions of interest the scan speed is reduced by a factor of 6 to obtain larger effective accumulation times per channel.

Figure 1 shows a typical Brillouin light scattering spectrum. The scattering geometry is shown in the inset of Fig. 2. At zero frequency the elastically scattered light, and at frequencies of $\pm(33-37) \mathrm{GHz}$ the ghost peaks are observed. In the regions of $\pm(6.5-22) \mathrm{GHz}$ the inelastically scattered signal is recorded. We observed the Damon-Eshbach mode, the first standing spin wave (volume mode), and a surface phonon (see Fig. 1). Figure 2 shows the observed dispersion curve of the Damon-Eshbach mode (squares) for the $2 / 2.2$ $\mu \mathrm{m}$ sample as a function of the in-plane wave vector, $q_{\|}$.

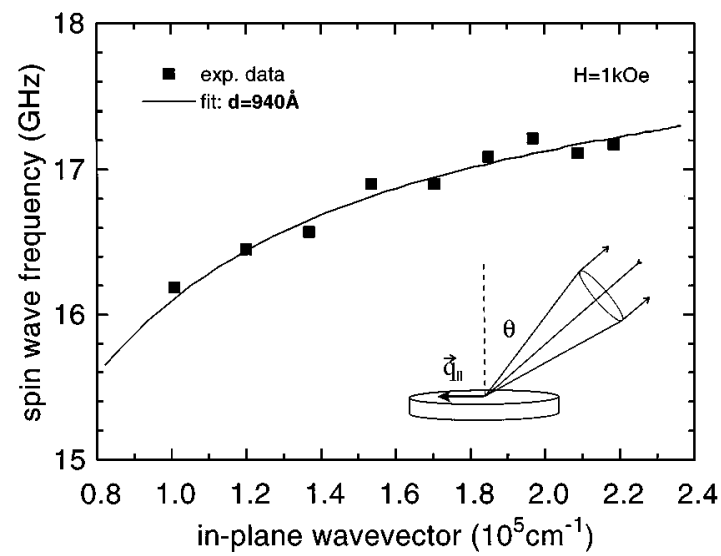

FIG. 2. Dispersion of the spin wave frequency for the $2 / 2.2 \mu \mathrm{m}$ sample as a function of the in-plane wave vector, $q_{\|}$. The scattering geometry is indicated in the inset. The squares are the experimental data and the solid line is a fit to the data.

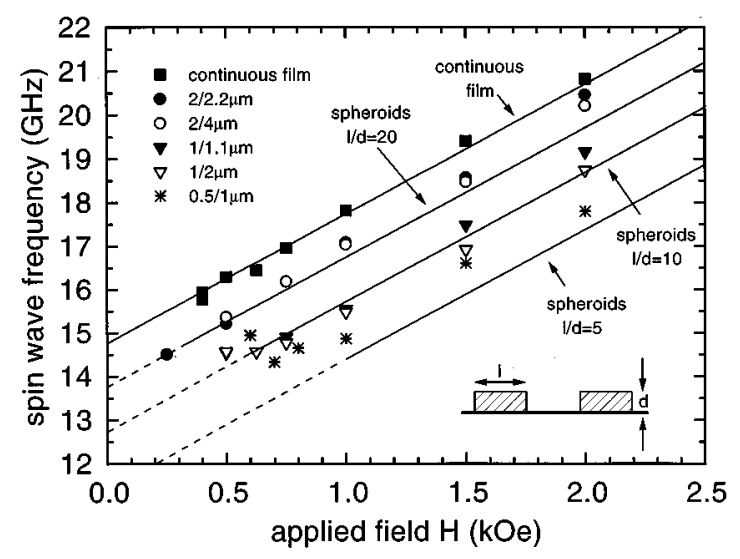

FIG. 3. Measured spin wave frequencies as a function of the strength of the applied external field, $H$. The symbols denote the measured data as indicated. The solid lines are fits to the data using aspect ratios of the spheroidal dot shapes as indicated in the figure.

The solid line is a fit using Eq. (1) with the thickness $d$ of the dots as a fit parameter. The fitted value of $d=940 \AA$ is in very good agreement with the nominal thickness of $1000 \AA$. No indications of a mode quantization due to the finite size of the dots, i.e., a lateral confinement of the spin wave modes, as reported for magnetic bars by Gurney et al.,,$^{5}$ or of spin wave zone folding effects as predicted by Kolodin et $a .^{6}$ have been observed for the investigated samples.

We have investigated in detail the dependence of the spin wave frequencies on the strength of the external field. Figure 3 shows the results for the $1000-\AA$-thick films. As obtained from the data displayed in Fig. 3 the spin wave frequencies are mostly sensitive to the dot diameter and to a much lesser degree to the dot separation. The solid lines are model calculations using Eq. (1) and calculating the demagnetizing factor, $N$, assuming spheroidally shaped dots with diameter $l$ to thickness $d$ ratios as indicated in the figure. A fairly good agreement between the measured and the calculated values is achieved for the data of spin wave frequencies larger than $14.5 \mathrm{GHz}$, where the dots are magnetically saturated. For smaller values, i.e., for $H<1 \mathrm{kOe}$ for the $l=0.5$ $\mu \mathrm{m}$ dots, and $H<0.6 \mathrm{kOe}$ for the $l=1.0 \mu \mathrm{m}$ dots, a breakdown of a uniform island magnetization is observed.

Next, of particular interest is the determination of coupling between the individual magnetic dots. In our data no coupling could be observed, apart from the dot arrays with the smallest dot separation of $0.1 \mu \mathrm{m}$. These data are displayed in Fig. 4 for the 1/1.1 $\mu \mathrm{m}$ sample and, as a reference, for the $1 / 2 \mu \mathrm{m}$ sample as a function of the in-plane direction of the external field of $1 \mathrm{kOe}$, measured against a reference direction, which is the [10]-direction of the dot array. The fourfold symmetry in the spin wave data of the $1 / 1.1 \mu \mathrm{m}$ sample is clearly observed. The data are analyzed writing the free in-plane anisotropic coupling energy as

$$
F=K^{(4)} \cdot \sin ^{2} \Phi \cos ^{2} \Phi
$$

with $\Phi$ the angle between the direction of magnetization, which is the same in each individual dot, and the [01]direction, and $K^{(4)}$ the in-plane anisotropy constant. From a model fit (solid line in Fig. 4) we obtained $K^{(4)}=-(1.4$ 


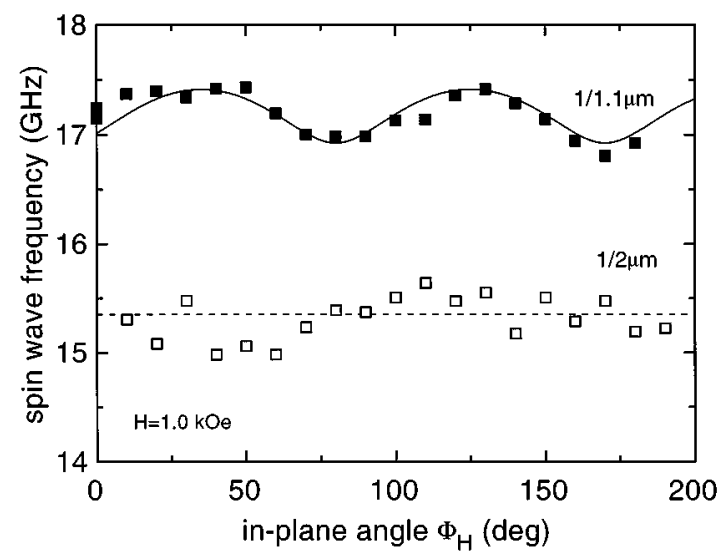

FIG. 4. Dependence of the spin wave frequencies from the in-plane direction of the applied field for the $1 / 1.1 \mu \mathrm{m}$ sample, and, for comparison, for the $1 / 2 \mu \mathrm{m}$ sample. The solid line is a fit for the data. For clarity the data of the $1 / 1.1 \mu \mathrm{m}$ sample is shifted in frequency by $2 \mathrm{GHz}$.

$\pm 0.3) \cdot 10^{5} \mathrm{erg} / \mathrm{cm}^{3}$. The easy axis is along the pattern diagonal, i.e., the [11]-axis. Measurements at an applied field of 2 kOe yield a similar behavior, but with a smaller value of $K^{(4)}=-(0.74 \pm 0.30) \cdot 10^{5} \mathrm{erg} / \mathrm{cm}^{3}$. Hysteresis loops measured by the magneto-optic Kerr effect show for the 1/1.1 $\mu \mathrm{m}$ sample corresponding characteristic changes in the loop shapes as a function of the in-plane direction of the applied field as well.

The origin of the observed anisotropic coupling can be explained as follows. The distance of $0.1 \mu \mathrm{m}$ between the dots, confirmed by electron micrographs, is too large to provide for a significant direct electronic exchange interaction mechanism via conduction electrons or electron tunneling. The dipolar interaction between the magnetic dots also cannot account for the observed anisotropy due to the fourfold symmetry of the dot patterns, as long as the dots are magnetically completely saturated and the system can be described by a uniform magnetization vector. This is because in this case the dipolar energy can be expressed as a bilinear form of the components of the magnetization vector. Such an energy expression can only correspond to a uniaxial anisotropy, since in a bilinear form direction cosines appear quadratic in highest order. We propose that the measured anisotropic coupling observed for the sample with the smallest dot separation might be caused by the dipole-dipole interaction between residual unsaturated parts of the dots, since for this case the energy cannot be expressed as a bilinear form. The large observed decrease of the coupling anisotropy constant with increasing field corroborates this assumption. The demagnetizing fields corresponding to the diameter/thickness ratio of the dots are smaller than the maximum applied field, by which the anisotropic coupling is still observed. However, due to the nonellipsoidal form of the dots and due to surface magnetic anisotropies, the nonsaturated parts can persist up to fairly large fields. More work is needed to clarify details of the interaction.

In summary, using spin waves as a tool to investigate magnetic properties in dot arrays, the most striking effect is the demagnetizing effect of the individual dots. From fitting the spin wave dispersion curves the dot thicknesses obtained agree with the nominal thicknesses. A coupling between the magnetic dots is observed only for the smallest investigated dot separation of $0.1 \mu \mathrm{m}$ (dot periodicity $1.1 \mu \mathrm{m}$ ). It should be noted that the large dependence on the demagnetizing factor is due to the fact that the spin wave wavelength of $\approx 2000 \AA$ is comparable to the island sizes. A breakdown of the uniform island magnetization can be easily observed in the spin wave spectra. For a full description of the spin wave mode dispersion in these patterned structures model calculations taking the size and the shapes of the islands in detail into account are greatly needed.

The authors would like to thank O. Büttner and S. Riedling for performing the Kerr hysteresis loop measurements. Support by the HCM program of the European Community under contract No. CHRX-CT93-0316 is gratefully acknowledged.

${ }^{1}$ F. Rousseaux, D. Decanini, F. Carcenac, E. Cambril, M. F. Ravet, C. Chappert, N. Bardou, B. Bartenlian, and P. Veillet, J. Vac. Sci. Technol. B 13, 2787 (1995)

${ }^{2}$ B. Hillebrands and G. Güntherodt, in Ultrathin Magnetic Structures II, edited by B. Heinrich, and J. A. C. Bland (Springer, Heidelberg, 1994), p. 258.

${ }^{3}$ M. G. Cottam and D. J. Lockwood, Light Scattering in Magnetic Solids (Wiley, New York, 1986), p. 199.

${ }^{4}$ B. Hillebrands, Phys. Rev. B 41, 530 (1990).

${ }^{5}$ B. A. Gurney, P. Baumgart, V. Speriosu, R. Fontana, A. Patlac, T. Logan, and P. Humbert, Digest of the International Conference on Magnetic Films and Surfaces, Glasgow, 1991.

${ }^{6}$ P. A. Kolodin and B. Hillebrands, J. Magn. Magn. Mater. (in press). 